\title{
Dietary Intake of Immature Citrus tumida Hort. ex Tanaka Peels Suppressed Body Weight Gain and Fat Accumulation in a Mouse Model of Acute Obesity
}

\author{
Mizuho SATO ${ }^{1,2}$, Tatsuhiko Goto ${ }^{1,3, *}$, Eiichi INOUE ${ }^{1,2,3}$, \\ Yuji Mryaguchi ${ }^{1,2,3}$ and Atsushi ToyodA ${ }^{1,2,3, * *}$ \\ ${ }^{1}$ College of Agriculture, Ibaraki University, Ami, Ibaraki 300-0393, Japan \\ ${ }^{2}$ United Graduate School of Agricultural Science, Tokyo University of Agriculture and Technology, \\ Fuchu, Tokyo 183-8509, Japan \\ ${ }^{3}$ Ibaraki University Cooperation between Agriculture and Medical Science (IUCAM), \\ Ami, Ibaraki 300-0393, Japan \\ (Received February 13, 2018)
}

\begin{abstract}
Summary Citrus fruits have several potential benefits for maintaining our health. In this study, we investigated the anti-obesity effects of immature Citrus tumida hort. ex Tanaka (C. tumida) peels using an acute obesity mice model. C57BL/6J male mice were divided into 2 groups; HFD-LL subjected to a high fat diet (HFD) and constant light exposure (LL), and HFDC-LL subjected to a HFD containing immature peel powder of C. tumida $(5 \% \mathrm{w} / \mathrm{w})$ and LL. Dietary ingestion of immature $C$. tumida peels significantly suppressed body weight gain following decreased epidydimal, perirenal, and subcutaneous fat weights. Blood levels of triglyceride and total cholesterol in the HFDC-LL were significantly lower than those in the HFD-LL group; however, there was no significant difference in food or calorie intake between the 2 groups. These results suggested that immature $C$. tumida peels have a beneficial effect on the prevention of obesity and metabolic syndrome via its biochemical activities of lipid metabolism.
\end{abstract}

Key Words obesity, Citrus tumida hort. ex Tanaka, high fat diet, mouse

Recently, more than 600 million adults have been known to suffer from obesity, and there are about $2 \times 10^{9}$ overweight people worldwide (http://www.who. int/mediacentre/factsheets/fs311/en/). Furthermore, cardiovascular disease, malignant neoplasm, diabetes, stroke, and depression are current major world health problems (1). These health problems are related to the increased consumption of energy-dense foods such as high fat diets, that will be a global issue in the next generation (http://www.who.int/mediacentre/factsheets/ fs $311 / \mathrm{en} /$ ). Feasible prevention and treatment strategies for obesity and obesity-induced health problems are essential for humans to maintain their quality of life and reduce world health expenditure.

A common strategy for reducing obesity is energy restriction combined with adequate exercise, and this strategy has been shown consistently to reduce obesity in large, randomized, controlled studies (2). Anti-obesity drugs can be divided into 5 categories: central appetite suppressants, digestion and absorption blockers, metabolic promoters, obesity gene product inhibitors, and other drugs for the treatment of obesity (3). However, weight loss due to conventional anti-obesity drugs has some side effects, such as drug dependence and insomnia

* Present address: Obihiro University of Agriculture and Veterinary Medicine, Obihiro, Hokkaido 080-8555, Japan

** To whom correspondence should be addressed.

E-mail: atsushi.toyoda.0516@vc.ibaraki.ac.jp
(4). Therefore, authors of recent studies have focused on the anti-obesity effect of foods with fewer side effects (5).

Appropriate animal models of obesity are necessary to evaluate the anti-obesity effect of food. Various obese models, such as mice subjected to a high fat diet (HFD) and genetic mutation, have been developed so far (6); however, developing these models can be time-consuming (7). Recently, researchers revealed that nighttime light exposure increases body weight and fat following short-term increased food intake (8). Furthermore, light exposure at night changes the insulin secretion patterns and promotes obesity (9). The obesity model created by light exposure at night reflects the health problems of night workers $(10,11)$.

Citrus is a popular fruit with various health benefits (12). Citrus depressa Hayata extract prevents obesity caused by HFD (13). Recently, we have taken an interest in Citrus tumida hort. ex Tanaka (C. tumida), which is a local citrus found around Mt. Tsukuba in Ibaraki Prefecture, Japan (14). However, the potential health benefits of $C$. tumida have not been investigated yet. In this study, we focused on the anti-obese activity of immature C. tumida peels because these peels highly contain citrus flavonoids, such as nobiletin. Therefore, we evaluated the anti-obese functions of this peel using an acute obesity mice model subjected to HFD under constant light exposure. 
(A)

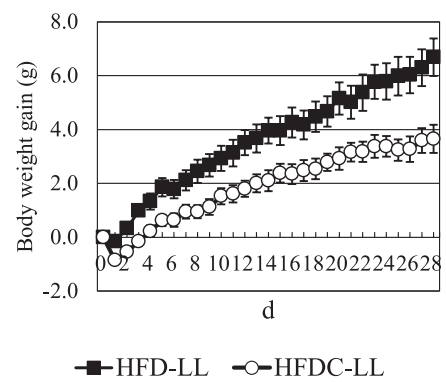

(C)

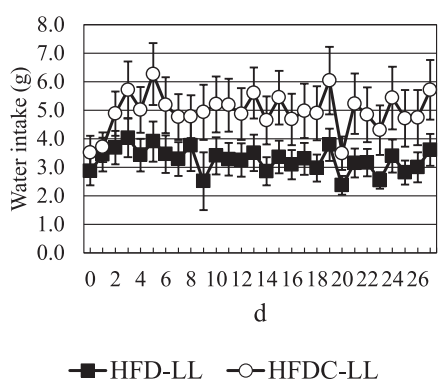

(B)

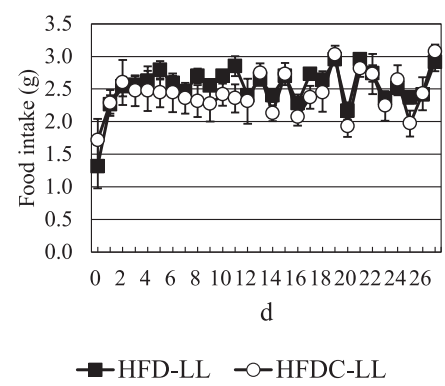

(I) - Effect of Citrus tumida (C. tumida) on general physiological features in mice under high-fat (HFD) and (LL). Temporal changes in body weight gain (A), food intake (B), and water intake (C) in mice in the HFD with LL (HFD-LL group; $n=7$ ) and HFD and immature $C$. tumida peel powder $5 \% \mathrm{w} / \mathrm{w}$ with LL (HFDC-LL group; $n=8$ ) are shown. Data are presented as mean \pm standard error of the mean.

\section{METHODS}

Experimental animal and protocols. All procedures of animal experiments were reviewed and approved by the guidelines of the Animal Care and Use Committee of Ibaraki University (Ibaraki, Japan), which conformed to the Ministry of Education, Culture, Sports, Science and Technology, Japan (Notification, No. 71).

Male C57BL/6JJmsSlc (B6) mice (7 wk of age) were purchased from Japan SLC, Inc. (Shizuoka, Japan), and housed individually in an ambient temperature of $23 \pm 1{ }^{\circ} \mathrm{C}$ with water and food ad libitum. After the acclimation period ( $1 \mathrm{wk}$ ) following purchase, all mice were housed under 12-h light: 12-h dark (LD) conditions and fed with a semi-purified diet powder (7\% fat, $17.9 \%$ protein, $60.6 \%$ carbohydrate) (AIN-93G, Oriental Yeast Co., Ltd., Tokyo, Japan). The 15 mice were then divided into 2 body weight-matched groups in each experiment. Seven mice were fed with HFD powder $(62.2 \%$ fat, $18.2 \%$ protein, $19.6 \%$ carbohydrate) (HFD-60, Oriental Yeast), while the other 8 mice were fed with HFD-60+immature C. tumida 5\% (w/w) peel powder (HFDC). Experiments were conducted for $4 \mathrm{wk}$ under the 24-h light (LL) conditions. Body weight, food intake, and water intake were measured daily. At the end of the experiments, the mice fasted for $3 \mathrm{~h}$ before blood samples were collected in tubes via their carotid arteries. Serum was separated from the blood by centrifugation at $1,000 \times g$ for $15 \mathrm{~min}$ at $4^{\circ} \mathrm{C}$. After blood collection, the mice were sacrificed by cervical dislocation and liver and adipose tissues, such as epidydimal, perirenal, and subcutaneous fat, were dissected. These tissues were weighed. Serum was quickly frozen by liquid nitrogen and stored at $-80^{\circ} \mathrm{C}$ until analysis.

Preparation of immature C. tumida peel powder. Immature $C$. tumida was harvested at an orchard in the eastern foothills of Mt. Tsukuba, Ibaraki Prefecture, Japan, in October 2014. Peels containing both outer orange and inner white layers were manually collected and freeze-dried with a freeze-dryer (FDU-1110, Tokyo Rikakikai, Tokyo, Japan). The dried peels were powdered with a centrifugal mill (ZM-1, Retsch Technology GmbH, Haan, Germany). Peel powder was stored at room temperature $\left(23-26^{\circ} \mathrm{C}\right)$ until use. The nutritional components of immature $C$. tumida peel powder were analyzed by the Japan Food Research Laboratories (Tokyo, Japan). The immature $C$. tumida peel $(100 \mathrm{~g})$ contains calorie (275 kcal), moisture (2.9 g), protein (7.4 g), fat (2.7 g), ash (4.9 g), carbohydrate (82.1 g), sugar (28.4 g), dietary fiber $(53.7 \mathrm{~g})$, galacturonic acid $(12.2 \mathrm{~g})$, and sodium (4.3 mg).

Serum biochemical assay. Concentrations of serum components, including total protein (TP), albumin (ALB), triglyceride (TG), total cholesterol (T-CHO), high density lipoprotein-cholesterol (HDL-C), glucose (GLU), aspartate aminotransferase (AST), alanine aminotransferase (ALT), lactate dehydrogenase (LDH), $\gamma$-glutamyltransferase $(\gamma$-GTP), total bilirubin (T-BIL), blood urea nitrogen (BUN), creatinine (CRE), sodium (Na), potassium $(\mathrm{K})$, chlorine $(\mathrm{Cl})$, calcium (Ca) and inorganic phosphorus (IP) were determined using the Hitachi 7180 auto analyzer (Hitachi, Tokyo, Japan) in the Nagahama Life Science Laboratory (Shiga, Japan). TP and ALB were analyzed by the Biuret and bromocresol green (BCG) 
Table 1. Effects of dietary intake of C. tumida peel on the components of blood serum.

\begin{tabular}{lccc}
\hline & HFD-LL & HFDC-LL & $p$-value \\
\hline TP $(\mathrm{g} / \mathrm{dL})$ & $5.8 \pm 0.1$ & $5.3 \pm 0.1$ & $p<0.01$ \\
ALB $(\mathrm{g} / \mathrm{dL})$ & $3.8 \pm 0.1$ & $4.0 \pm 0.2$ & $\mathrm{~ns}$ \\
TG $(\mathrm{mg} / \mathrm{dL})$ & $36.6 \pm 5.1$ & $24.9 \pm 3.0$ & $p<0.05$ \\
T-CHO (mg/dL) & $170.1 \pm 11.6$ & $128.3 \pm 9.4$ & $p<0.01$ \\
HDL-C (mg/dL) & $93.7 \pm 5.8$ & $79.8 \pm 8.2$ & $p<0.1$ \\
GLU (mg/dL) & $127.4 \pm 10.5$ & $104.1 \pm 11.0$ & $p<0.1$ \\
AST (IU/L) & $251.3 \pm 34.3$ & $347.8 \pm 71.9$ & $\mathrm{~ns}$ \\
ALT (IU/L) & $33.7 \pm 2.1$ & $48.9 \pm 14.7$ & $\mathrm{~ns}$ \\
LDH (IU/L) & $1,636.9 \pm 173.4$ & $1,830.6 \pm 210.5$ & $\mathrm{~ns}$ \\
$\gamma-\mathrm{GTP}(\mathrm{IU} / \mathrm{L})$ & $7.3 \pm 0.9$ & $9.0 \pm 1.4$ & $\mathrm{~ns}$ \\
T-BIL (mg/dL) & $0.05 \pm 0.01$ & $0.07 \pm 0.01$ & $\mathrm{~ns}$ \\
BUN (mg/dL) & $32.9 \pm 1.7$ & $29.0 \pm 1.6$ & $p<0.1$ \\
CRE (mg/dL) & $0.18 \pm 0.02$ & $0.20 \pm 0.01$ & $\mathrm{~ns}$ \\
Na (mEq/L) & $154.4 \pm 1.1$ & $152.9 \pm 0.8$ & $\mathrm{~ns}$ \\
K (mEq/dL) & $10.3 \pm 0.4$ & $10.2 \pm 0.5$ & $\mathrm{~ns}$ \\
Cl (mEq/dL) & $99.1 \pm 1.1$ & $96.5 \pm 1.6$ & $\mathrm{~ns}$ \\
Ca (mg/dL) & $12.1 \pm 1.5$ & $15.0 \pm 2.3$ & $\mathrm{~ns}$ \\
IP (mg/dL) & $9.6 \pm 0.5$ & $8.7 \pm 0.3$ & $p<0.01$ \\
\hline
\end{tabular}

Data are expressed mean \pm SE.

methods, respectively. BUN, CRE, IP, T-CHO, TG, T-BIL, and GLU were analyzed by the enzymatic method. Na, $\mathrm{K}$, and $\mathrm{Cl}$ were analyzed by the electrode method. AST, ALT, LDH, and $\gamma$-GTP were analyzed by the Japan Society of Clinical Chemistry (JSCC) transferable method. $\mathrm{Ca}$ and HDL-C were analyzed by the $o$-cresolphthalein complexone (OCPC) and accelerator selective detergent methods, respectively.

Statistical analysis. Statistical testing for body weight gain, food intake, and water intake were performed using two-way (repeated measure) analysis of variance (ANOVA) for the effects on food, time, and their interaction. Serum components and adipose weight were determined using Student's t-test. Data were analyzed with Excel Toukei 2006 for Windows (Social Survey Research Information Co. Ltd., Tokyo, Japan). The threshold of statistical significance was set to $p<0.05$. The trend for statistical significance was set to $p<0.1$.

\section{RESULTS}

Body weight gain, food intake, water intake, and total calorie intake

According to the two-way repeated measures ANOVA, a significant effect of $C$. tumida on body weight gain was observed for $4 \mathrm{wk}$ in the feeding period $\left(F_{1,364}=11.38\right.$, $p<0.01)$. The average body weight gain in peel-fed mice (HFDC-LL) was lower than that of mice subjected to HFD-LL (Fig. 1A). However, there was no significant difference in the food intake between the 2 groups for these $4 \mathrm{wk}\left(F_{1,351}=0.35, p>0.1\right.$; Fig. 1B). Furthermore, there was no significant difference in the water intake $\left(F_{1,351}=1.79, p>0.1\right.$; Fig. $\left.1 C\right)$ and cumulative total calorie intake (HFD-LL, 343.0 $\pm 11.4 \mathrm{kcal}$ vs. HFDCLL, $342.9 \pm 26.0 \mathrm{kcal}, \quad p>0.1)$, or cumulative total water intake (HFD-LL, $100.5 \pm 13.4 \mathrm{~g}$ vs. HFDC-LL,
$138.6 \pm 24.3 \mathrm{~g}, p>0.1$ ) between the 2 groups.

Body weight, liver and body fat weights

The body weight of HFDC-LL mice was significantly lower than that of HFD-LL mice $(30.3 \pm 1.1 \mathrm{~g}$ vs. $27.2 \pm 0.6 \mathrm{~g}, p<0.01)$. There was no significant difference in the liver weight (\% body weight) between the HFD-LL and HFDC-LL groups $(3.05 \pm 0.06 \%$ vs. $3.34 \pm 0.21 \%$, respectively, $p>0.1$ ). The body fat weights of HFDC-LL mice were significantly lower than those of HFD-LL mice. The epidydimal, perirenal, and subcutaneous fat weights (\% body weight) were higher in the HFD-LL group $(3.22 \pm 0.57 \%, 1.47 \pm 0.30 \%$, and $0.59 \pm 0.09 \%$, respectively) compared to the HFDC-LL group $(1.75 \pm 0.30 \%, p<0.05 ; 0.61 \pm 0.16 \%, p<0.05$; and $0.29 \pm 0.05 \%, p<0.01$, respectively).

Biochemical assays for serum components

We measured 18 serum components as shown in Table 1. The TP, T-CHO, and TG concentrations in serum of HFDC-LL mice were significantly decreased compared to those of HFD-LL mice (TP: $p<0.01$; T-CHO: $p<0.01$; TG: $p<0.05)$. The HDL-C, GLU, BUN, and IP concentrations in serum of HFDC-LL mice were slightly reduced compared to those of HFD-LL mice (HDL-C: $p=0.09$; GLU: $p=0.09$; BUN: $p=0.06$; IP: $p=0.09$ ).

\section{DISCUSSION}

In this study, we found an anti-obesity effect in the peel of immature $C$. tumida using a mouse model of acute obesity. The HFDC-LL mice showed significantly suppressed body weight gain and body fat compared to HFD-LL mice (Fig. 1A). However, there were no significant differences in total food and calorie intakes between HFDC-LL and HFD-LL mice. Previously, it has been reported that dietary intake of grapefruit (Citrus paradisi) (15), Citrus unshiu (16), Citrus bergamia (17), Cit- 
rus sunki (18), Citrus sudachi (19), and Citrus junos (20) suppressed body weight, body fat, blood TG, and T-CHO in obese mouse models. These studies showed that hesperidin, neohesperidin, nobiletin, tangeretin, and naringin have critical roles in the effects $(15-20)$. The peel of immature C. tumida decreased blood TG and T-CHO similarly to other citrus fruits. In addition, as with other citrus fruits, C. tumida had a tendency to suppress blood glucose levels (Table 1). Previously, Sakai et al. reported that hesperidin (340 mg), nobiletin (34 mg), tangeretin (34 mg), eriocitrin $(2.8 \mathrm{mg})$, neoeriocitrin $(5.8 \mathrm{mg})$, narirutin $(13.2 \mathrm{mg})$, and sinensetin $(2.8 \mathrm{mg})$ were contained in $100 \mathrm{~g}$ of fresh peel of immature C. tumida (21). They also claimed that the peel of immature $C$. tumida contains more hesperidin, nobiletin, and tangeretin than the peel of Citrus unshiu (21).

Several citrus flavonoids showed significant anti-obesity effects: reduced plasma lipid concentration, body fat, and body weight (22).

Hesperidin is one of the most popular flavonoids contained in various citrus fruits (23). Previous researchers described how hesperidin inhibits liver cholesterol synthesis, 3-hydroxy-3-methylglutaryl-coenzyme A, and acyl-CoA cholesterol acyltransferase $(24,25)$. Furthermore, C. tumida contains polymethoxylated flavones, such as nobiletin and tangeretin (26). It was suggested that nobiletin induces the activation of HIB1B cells, a brown adipocyte cell line derived from a mouse brown fat tumor and mitochondrial biogenesis of 3T3-L1 white adipocytes (27). In addition, chronic nobiletin suppressed body fat and plasma TG in an obese mice model by suppressing PPAR $\gamma$, fatty acid synthase, and sterol regulatory element-binding protein-1c in the liver (28). To summarize, nobiletin promotes energy metabolism and inhibits fatty acid synthesis. Furthermore, tangeretin improves insulin resistance by suppressing the expression of obesity-induced inflammatory cytokines in the coculture of 3T3-L1 adipocytes and RAW 264.7 cells (29). Constant light exposure increased short-term body fat accumulation because of changed feeding timing, pattern of insulin secretion, and circadian rhythm $(8,9)$. Interestingly, previous researchers reported that nobiletin regulates circadian rhythm and suppresses body fat accumulation and weight gain $(30,31)$. Therefore, these flavonoids in the peel of immature C. tumida may be involved in suppressing body weight and fat in this acutely obese mouse model.

Citrus peel contains high levels of dietary fibers showing anti-obese effects (32). Actually, the consumption of a $10 \%$ pectin diet significantly suppressed body fats and weights in mice (33). However, 2.5\% inulin and 10\% inulin feeding were unable to control rat weight (34). In addition, according to Isken et al., mice fed soluble fiber showed an increase in body weight (35). The peel of immature C. tumida powder (100 g) contains total dietary fiber $(53.7 \mathrm{~g})$ and galacturonic acid (12.2 g). Therefore, HFDC mice were fed approximately $2.7 \%$ of dietary fiber from the immature C. tumida peel powder, which might impact body weight and fat accumulation.

HFDC-LL mice showed lower TP levels in the blood compared to HFD-LL mice (Table 1). However, the ALB level in the blood did not change. We need to revalidate the TP and ALB in the blood.

In conclusion, we discovered the anti-obesity effect of immature $C$. tumida peel using a mouse model of acute obesity subjected to HFD under a constant light environment. In the future, a comprehensive analysis, such as metabolome and transcriptome, should be conducted to elucidate the molecular mechanism of the anti-obese action of C. tumida and identify critical components of the peel that contribute to this action.

\section{Acknowledgments}

We thank Dr. T. Okayama and Ms. H. Shimonishi (Ibaraki University, Japan) for their valuable experimental and technical assistance. We also thank Ms. H. Suda and Mr. T. Shiratori (Ibaraki University, Japan) for their assistance with the initial part of the study. This research was supported in part by Ibaraki University Cooperation between Agriculture and Medical Science (IUCAM) (The MEXT, Japan), Prioritised Research (Ibaraki University, Japan), and the Council for Science, Technology and Innovation (CSTI) under the Cross-ministerial Strategic Innovation Promotion Program (SIP) "Technologies for creating next-generation agriculture, forestry, and fisheries" (Bio-oriented Technology Research Advancement Institution, NARO) (The Cabinet Office, Japan).

\section{REFERENCES}

1) GBD 2013 DALYs and HALE Collaborators. 2016. Global, regional, and national disability-adjusted life years (DALYs) for 306 disease and injuries and healthy life expectancy (HALE) for 188 countries, 1990-2013: quantifying the epidemiological transition. Lancet $\mathbf{3 8 6}$ : 2145-2191.

2) Teixeira PJ, Margues MM. 2017. Health behavior change for obesity management. Obes Facts 10: 666-673.

3) Apovian CM, Aronne LJ, Bessesen DH, McDonnell ME, Murad MH, Pagotto U, Ryan DH, Still CD. 2015. Pharmacological management of obesity: an endocrine Society clinical practice guideline. J Clin Endocrinol Metab 100: 342-362.

4) Dietrich MO, Horvath TL. 2012. Limitations in antiobesity drug development: the critical role of hungerpromoting neurons. Nat Rev Discov 11: 675-691.

5) Kishino E, Ito T, Fujita K, Kiuchi Y. 2006. A mixture of the Salacia reticulata (Kotala himbutu) aqueous extract and cyclodextrin reduces the accumulation of visceral fat mass in mice and rats with high-fat diet-induced obesity. J Nutr 136: 433-439.

6) Nakagawa T, Hu H, Zharikov S, Tuttle KR, Short RA, Glushakova O, Ouyang X, Feig DI, Block ER, HerreraAcosta J, Patel JM, Johnson RJ. 2006. A causal role for uric acid in fructose-induced metabolic syndrome. Am J Physiol Renal Physiol 290: F625-631.

7) Kinzig KP, Scott KA, Hyun J, Bi S, Moran TH. 2005. Altered hypothalamic signaling and responses to food deprivation in rats fed a low-carbohydrate diet. Obes Res 13: 1672-1682.

8) Fonken LK, Workman JL, Walton JC, Weil ZM, Morris JS, Haim A, Nelson RJ. 2010. Light at night increases body mass by shifting the time of food intake. Proc Natl Acad Sci USA 107: 18664-18669. 
9) Fonken LK, Lieberman RA, Weil ZM, Nelson RJ. 2013. Dim light at night exaggerates weight gain and inflammation associated with a high-fat diet in male mice. Endocrinology 154: 3817-3825.

10) Kettner NM, Voicu H, Finegold MJ, Coarfa C, Sreekumar A, Putluri N, Katchy CA, Lee C, Moore DD, Fu L. 2016. Circadian homeostasis of liver metabolism suppresses hepatocarcinogenesis. Cancer Cell 30: 909-924.

11) Obayashi K, Saeki K, Iwamoto J, Okamoto N, Tomioka K, Nezu S, Ikada Y, Kurumatani N. 2013. Exposure to light at night, nocturnal urinary melatonin excretion, and obesity/dyslipidemia in the elderly: A cross-sectional analysis of the HEIJO-KYO study. J Clin Endocrinol Metab 98: 337-344.

12) Mulvihill EE, Burke AC, Huff MW. 2016. Citrus flavonoids as regulators of lipoprotein metabolism and atherosclerosis. Annu Rev Nutr 36: 275-299.

13) Lee YS, Cha BY, Saito K, Choi SS, Wang XX, Choi BK, Yonezawa T, Teruya T, Nagai K, Woo JT. 2011. Effects of a Citrus depressa Hayata (shiikuwasa) extract on obesity in high-fat diet-induced obese mice. Phytomedicine 18: 648-654.

14) Tanaka T. 1954. Species problem in Citrus: a critical study of wild and cultivated units of Citrus, based upon field studies in their native homes (Revisio aurantiacearum). JSPS 9: 139.

15) Chudnovskiy R, Thompson A, Tharp K, Hellerstein M, Napoli JL, Stahl A. 2014. Consumption of clarified grapefruit juice ameliorates high-fat diet induced insulin resistance and weight gain in mice. Plos One 9: e108408.

16) Kim GN, Shin MR, Shin SH, Lee AR, Seo BI, Kim MY, Kim TH, Noh JS, Rhee MH, Roh SS. 2016. Study of antiobesity effect through inhibition of pancreatic lipase activity of Diospyros kaki fruit and Citrus unshiu Peel. Biomed Res Int 2016: 1723042.

17) Lo Furno D, Graziano AC, Avola R, Giuffrida R, Perciavalle V, Bonina F, Mannino G, Cardile V. 2016. A Citrus bergamia extract decreases adipogenesis and increases lipolysis by modulating PPAR levels in mesenchymal stem cells from human adipose tissue. PPAR Res $\mathbf{2 0 1 6}$ 4563815 .

18) Kang SI, Shin HS, Kim HM, Hong YS, Yoon SA, Kang SW, Kim JH, Kim MH, Ko HC, Kim SJ. 2012. Immature Citrus sunki peel extract exhibits antiobesity effects by $\beta$-oxidation and lipolysis in high-fat diet-induced obese mice. Biol Pharm Bull 35: 223-230.

19) Kobayashi H, Mitani M, Minatogawa Y, Hayashi S, Nakamoto M, Shuto E, Nii Y, Sakai T. 2017. Extracts of citrus Sudachi peel attenuate body weight gain in C57BL/ 6 mice fed a high-fat diet. J Med Invest 64: 20-23.

20) Kim AH, Kim HJ, Ryu R, Han HJ, Han YJ, Lee MK, Choi MS, Park YB. 2016. A mixture of ethanol extracts of persimmon leaf and Citrus junos Sieb improves blood coagulation parameters and ameliorates lipid metabolism disturbances caused by diet-induced obesity in C57BL/6J mice. J Microbiol Biotechnol 26: 295-308.

21) Sakai S, Nakagawa, R. 2011. Kensan Nousanhin no Kinousei Seibun ni Kansuru Shiken Kenkyu (The studies on functional ingredients in the agricultural products from Ibaraki Prefecture). Bull Ind Technol Inst Ibaraki Pref 40.

22) Bravo L. 1998. Polyphenols: chemistry, dietary sources, metabolism, and nutritional significance. Nutr Rev 56: 317-333.

23) Iranshahi M, Rezaee R, Parhiz H, Roohbakhsh A, Soltani F. 2015. Protective effects of flavonoids against microbes and toxins: The cases of hesperidin and hesperetin. Life Sci 137: 125-132.

24) Chiba H, Uehara M, Wu J, Wang X, Masuyama R, Suzuki K, Kanazawa K, Ishimi Y. 2003. Hesperidin, a citrus flavonoid, inhibits bone loss and decreases serum and hepatic lipids in ovariectomized mice. J Nutr 133: 1892-1897.

25) Bok HS, Lee HS, Park BY, Bae KH, Son KH, Jeong TS, Choi MS. 1999. Plasma and hepatic cholesterol and hepatic activities of 3-hydroxy-3-methyl-glutaryl-CoA reductase and acyl CoA: Cholesterol transferase are lower in rats fed citrus peel extract or a mixture of citrus bioflavonoids. J Nutr 129: 1182-1185.

26) Kawaii S, Tomono Y, Katase E, Ogawa K, Yano M. 1999. Quantitation of flavonoid constituents in citrus fruits. $J$ Agric Food Chem 47: 3565-3571.

27) Lone J, Parray HA, Yun JW. 2017. Nobiletin induces brown adipocyte-like phenotype and ameliorates stress in 3T3-L1 adipocytes. Biochimie 146: 97-104.

28) Lee YS, Cha BY, Choi SS, Wang XX, Choi BK, Yonezawa T, Teruya T, Nagai K, Woo JT. 2013. Nobiletin improves obesity and insulin resistance in high-fat diet-induced obese mice. J Nutr Biochem 24: 156-162.

29) Shin HS, Kang SI, Ko HC, Park DB, Kim SJ. 2017. Tangeretin improves glucose uptake in a coculture of hypertrophic adipocytes and macrophages by attenuating inflammatory changes. Dev Reprod 21: 93-100.

30) He B, Nohara K, Park N, Park YS, Guillory B, Zhao Z, Garcia JM, Koike N, Lee CC, Takahashi JS, Yoo SH, Chen Z. 2016. The small molecule nobiletin targets the molecular oscillator to enhance circadian rhythms and protect against metabolic syndrome. Cell Metab 23: 610-621.

31) Shinozaki A, Misawa K, Ikeda Y, Haraguchi A, Kamagata M, Tahara Y, Shibata S. 2017. Potent effects of flavonoid nobiletin on amplitude, period, and phase of the circadian clock rhythm in PER2::LUCIFERASE mouse embryonic fibroblasts. PLoS One 12: e0170904.

32) Kim BS, Kim YM, Jae J, Watanabe C, Kim S, Jung SC, Kim SC, Park YK. 2015. Pyrolysis and catalytic upgrading of Citrus unshiu peel. Bioresour Technol 194: 312-319.

33) Adam CL, Thomson LM, Williams PA, Ross AW. 2015. Soluble fermentable dietary fibre (Pectin) decreases caloric intake, adiposity and lipidaemia in high-fat dietinduced obese rats. PloS One 10: e0140392.

34) Singh A, Zapata RC, Pezeshki A, Reidelberger RD, Chelikani PK. 2018. Inulin fiber dose-dependently modulates energy balance, glucose tolerance, gut microbiota, hormones and diet preference in high-fat-fed male rats. J Nutr Biochem 59: 142-152.

35) Isken F, Klaus S, Osterhoff M, Pfeiffer AF, Weickert MO. 2010. Effects of long-term soluble vs. insoluble dietary fiber intake on high-fat diet-induced obesity in C57BL/6 J mice. J Nutr Biochem 21: 278-284. 